\title{
Effective Nutritional Guidance for Obesity by Low Carbohydrate Diet (LCD)
}

Nakamura $\mathrm{T}^{1,2}, \underline{\text { Kawashima } \mathrm{T}^{1,2}}$, Dobashi $\mathrm{M}^{1,2}, \underline{\text { Narita }}^{1,2},{\underline{\text { Bando } \mathrm{H}^{2,3^{*}}}}$

${ }^{1}$ Nakamura Orthopedic Clinic and Anti-Aging Center, Kawanishi, Hyogo, Japan

2Japan Low Carbohydrate Diet Promotion Association (JLCDPA), Kyoto, Japan

${ }^{3}$ Tokushima University / Medical Research, Tokushima, Japan

Corresponding Author: Hiroshi Bando, MD, PhD, FACP

Address: Tokushima University /Medical Research, Nakashowa 1-61, Tokushima 770-0943, Japan.

Received date: 02 January 2019; Accepted date: 19 January 2019; Published date: 27 January 2019

Citation: Nakamura T, Kawashima T, Dobashi M, et al., "Effective Nutritional Guidance for Obesity by Low Carbohydrate Diet (LCD)", Asp Biomed Clin Case Rep, vol.2, no.s1: 16-21, 2019.

Copyright (c) 2019 Nakamura T, Kawashima T, Dobashi M, et al., This is an open access article distributed under the Creative Commons Attribution License, which permits unrestricted use, distribution, and reproduction in any medium, provided the original work is properly cited.

\section{Abstract}

Background: Authors have continued treating patients with obesity and metabolic syndrome by application of Low Carbohydrate Diet (LCD). Furthermore, we have continued presenting various reports about nutrition and educational guidance and developing LCD medically and socially through the activity of Japan LCD Promoting Association (JLCDPA).

Study Protocol: Subjects enrolled were 2773 patients with obesity who received nutritional guidance in our clinic. Methods include i) weight reduction program on the intervention of LCD and guidance by registered dietitian nutritionist (RDN), ii) LCD with $33 \%$ of carbohydrate, and iii) Practice of guidance including self-monitoring, lifestyle, motivation, determination for the goal, evaluation.

Results and Discussion: Nutritional guidance has been carried out on a regular basis by three RDNs. Weight reduction was: $10 \%$ or more was observed in $666(24.0 \%)$, and $5.0-9.9 \%$ was in 863 (31.2\%). In other words, the reduction of $5.0 \%$ or more was $55.2 \%$, and $3.0 \%$ or more was $71.4 \%$. Our nutritional guidance has been made as a manual for weight reduction program. It includes various factors, such as Self-Regulation Empowerment, cognitive behavioral therapy and motivational interviewing. These results would become basal and reference data for future research in the field of weight reduction.

\section{Keywords:}

Obesity; Low Carbohydrate Diet (LCD); Weight Reduction; Nutritional Guidance; Self-Regulation Empowerment Program (SREP); Registered Dietitian Nutritionist (RDN)

\section{Abbreviation:}

LCD: Low Carbohydrate Diet; T2DM: Type 2 diabetes mellitus; CR: Calorie Restriction; SREP: SelfRegulation Empowerment Program; RDN: Registered Dietitian Nutritionist; JLCDPA: Japan LCD Promoting Association 
Citation: Nakamura T, Kawashima T, Dobashi M, et al., "Effective Nutritional Guidance for Obesity by Low Carbohydrate Diet (LCD)", Asp Biomed Clin Case Rep, vol.2, no.s1: 16-21, 2019.

\section{Introduction}

There are three kinds of treatments for obesity and diabetes mellitus, which are diet, exercise, and medicine [1, 2]. Among them, diet therapy is the foundation of them. As a standard method for therapy, calorie restriction (CR) has long been known and practiced. Thereafter, a method called Low Carbohydrate Diet (LCD) was introduced by Atkins, Bernstein, and other investigators $[3,4,5]$. LCD gradually became recognized and spread in Western countries. This effect has been widely recognized, and LCD has been prevalent not only in the medical field but also in the health, education and social fields.

On the other hand, LCD in Japan was firstly reported by Ebe et al. who applied to diabetic patients, and Dr. Kamaike began to LCD [6]. Authors had opportunities to actually meet two doctors, discussed and understood the detail of LCD. After that, we have also started and expanded LCD in Japan, recognizing the effectiveness of LCD [7]. The effect was clarified more than we anticipated, and reliability for LCD has been spread [8]. Furthermore, we developed LCD by some textbooks and reports medically and socially [9].

As mentioned above, the authors have instructed a weight reduction program for many obese patients. The crucial matters are not merely the contents of the LCD program. It is more important how to explain the patient, to convince, to motivate and continue the meal pattern for a long time $[10,11]$. In this situation, the presence of registered dietitian nutritionist (RDN) close to the patient would be the key point. We have been able to continue fine guidance by RDNs over the years, and achieve successful results for weight reduction to many obese people. The aim of this study is the evaluation of our guidance for the weight reduction program, with the statistical data of many subjects. The detail of the results and discussion would be presented in this article.

\section{Study Protocol}

The subjects are patients with obesity and metabolic syndrome including diabetes, hypertension, and hyperlipidemia. Subjects were
2773 patients, who underwent medical treatment at our clinic and also received nutritional guidance and exercise therapy for the purpose of weight reduction. The background of the patients shows that the average age in the median was 64 years old, quartile of $25 \%$ and $75 \%$ was 55 and 71 years old, and average and the standard deviation was 62 and 12 years, respectively. These data mean that central $50 \%$ of the subjects were distributed between 55 years old and 71years old. The criteria of the inclusion were that the subjects were treated and advised at our clinic and that the diagnosis was obesity with body mass index (BMI) was more than $25 \mathrm{~kg} / \mathrm{m}^{2}$. It is the standard borderline in Japan. On the other hand, the criteria of the exclusion were the patient that was dropped out from our treatment and guidance within 4-8 weeks. However, there was actually no case of exclusion situation.

Concerning the method, there are three aspects in the following.

\section{Weight reduction:}

The body weight at first visit was taken as the former value. The weight when the body weight decreased and became stable after 6-12 months was regarded as the latter value. The decrease value between the former and the latter was determined, which was decreased weight $(\mathrm{kg})$. The reduction weight ratio (\%) was calculated from decreased weight / former value.

\section{Low Carbohydrate Diet (LCD):}

As to LCD, we have taken advantages of several types of research meal. There is superLCD with $12 \%$ carbohydrate, standard-LCD with $26 \%$ carbohydrate, petite-LCD with $40 \%$ carbohydrate, 33\% anti-aging-LCD with 33\% carbohydrate. We have instructed continuation of LCD for the patients, in which the ratio of the carbohydrate included was scheduled to be $33 \%$. In the clinic, three RDNs have vigorously instructed a number of times so that LCD can be continued with the supportive instruction.

\section{Practice of Nutritional Guidance:}

In our weight reduction protocol, there were 


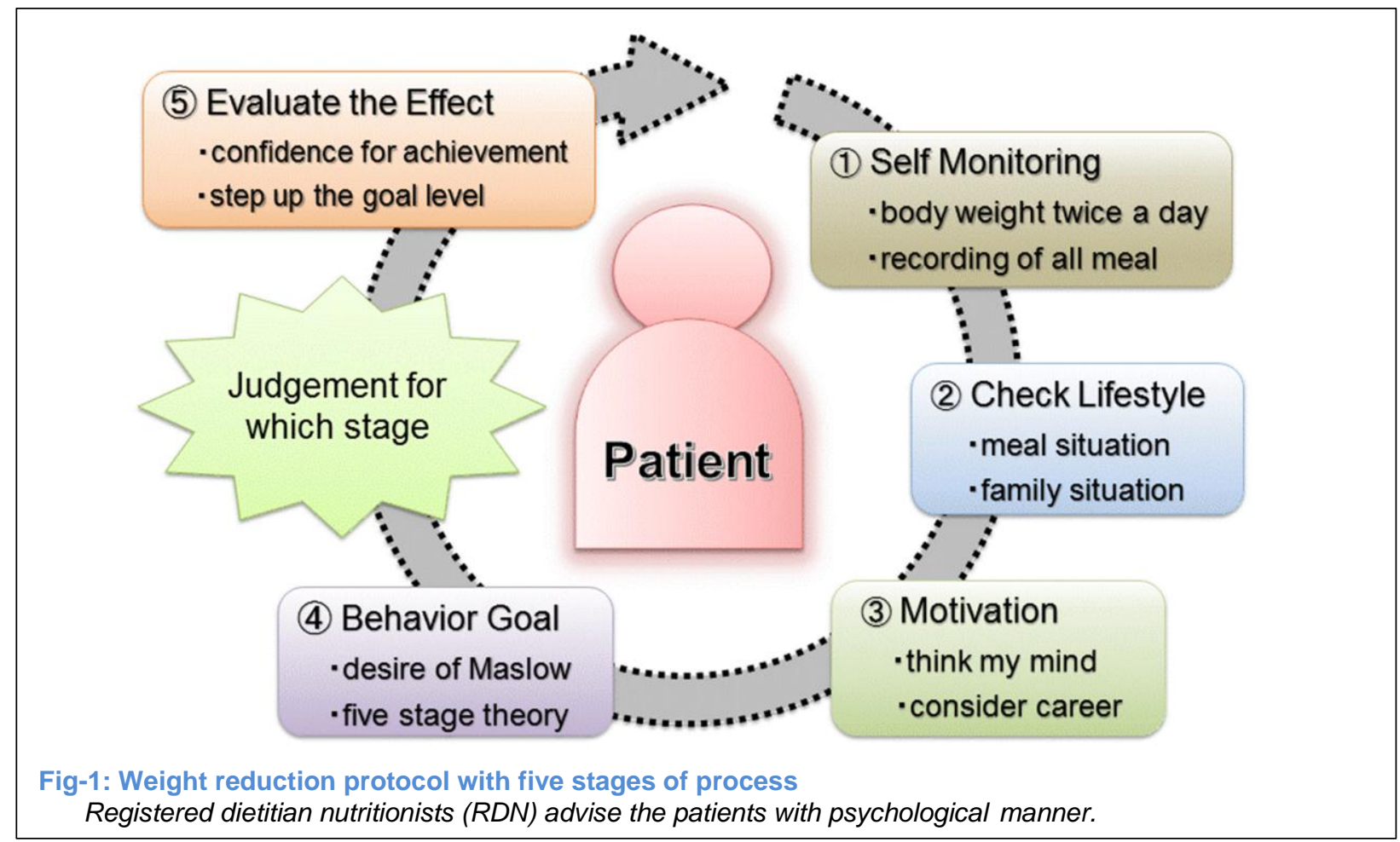

five stages of the process for the patients from RDNs (Fig-1).

I. Self-monitoring, weight measurement, description of graphing (2 times/day), recording meals.

II. Check lifestyle, diet form (self-catering, facilities), and family situation.

III. Motivation as a confirmation of the subject 's thoughts.

IV. Determination for the goal such as Maslow's five-step desire (short-term, medium-term, long-term goal) and the timing such as contemplation period, preparation period, etc.

V. Real feeling and evaluation of the effect (Health behavior, self-confidence, step up the target)

This guidance would be provided to the patients by the RDNs in our clinic.

\section{Results and Discussion:}

Nutrition guidance has been carried out on a regular basis by three RDNs along to the five steps as a rule. The results of 2773 cases are shown in Fig-2. There was a decrease of weight in the following: Weight reduction of $10 \%$ or more was observed in 666 (24.0\%), and reduction of $5.0-9.9 \%$ was in $863(31.2 \%)$. In other words, the reduction of $5.0 \%$ or more was observed in $55.2 \%$, and reduction of $3.0 \%$ or more was $71.4 \%$.

This study included the data more than 2700 cases and it seems to be one of the meaningful mass studies in Japanese. The place studied is not an urban area or a remote area, but an average local city close to Kobe city. The patient is not a specific subject visiting a university hospital, but a patient with a usual average background in the city. Consequently, the current result is considered to represent generally average data and situation in Japan.

In comparison with the weight reduction degree, there was a report of the Dietary Intervention Randomized Controlled Trial (DIRECT) Group. The mean values of weight loss in the three diet regimens over two years with three diet are as follows: Low-fat group 2.9 $\mathrm{kg}$, Mediterranean-diet group $4.4 \mathrm{~kg}$ and LCD group $4.7 \mathrm{~kg} \quad(p<0.001$ for the interaction between diet group and time) [12]. When calculated from subjects with the completion of the intervention for 2 years, mean weight reduction was $3.3 \mathrm{~kg}, 4.6 \mathrm{~kg}$, and $5.5 \mathrm{~kg}$, 
Citation: Nakamura T, Kawashima T, Dobashi M, et al., "Effective Nutritional Guidance for Obesity by Low Carbohydrate Diet (LCD)", Asp Biomed Clin Case Rep, vol.2, no.s1: 16-21, 2019.

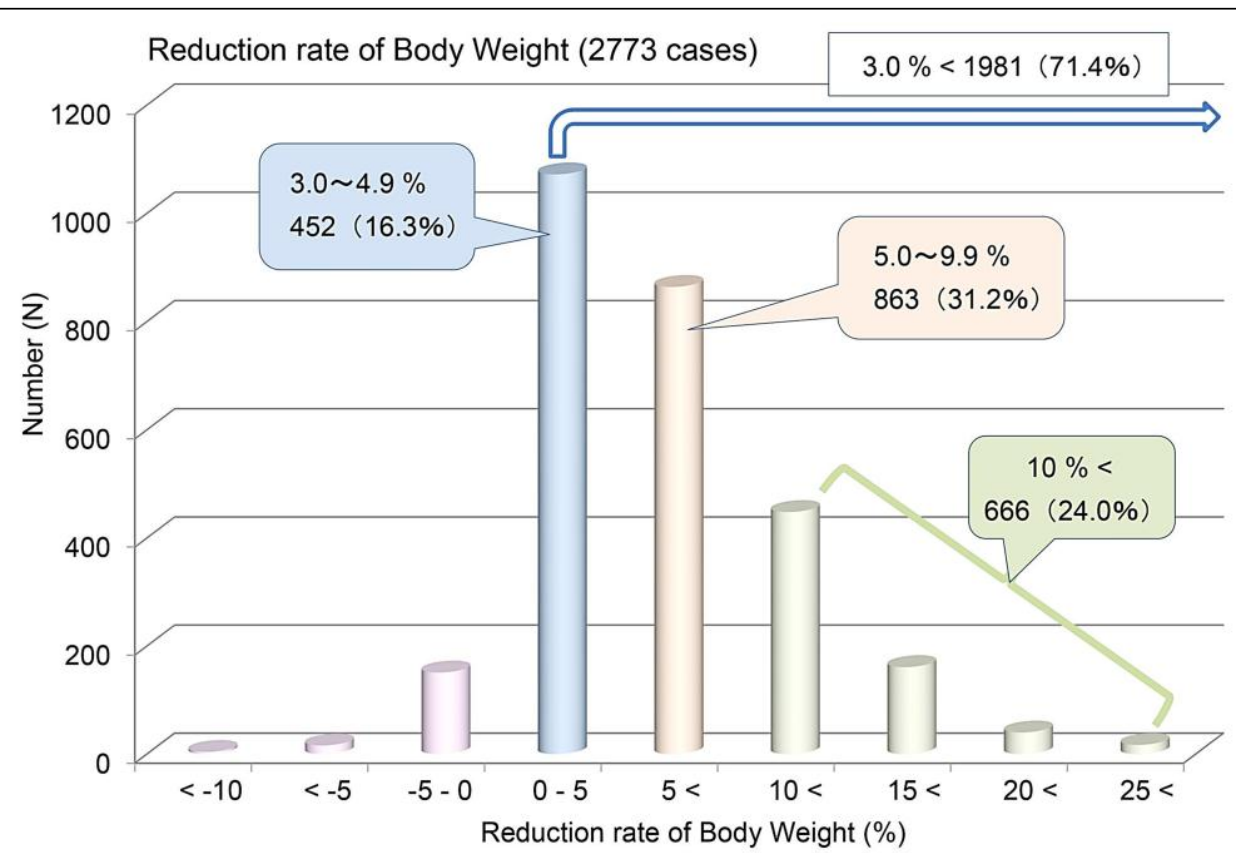

Fig-2: Reduction rate of body weight in the subject

Weight reduction was found out of 2773 cases as follows: $10 \%$ or more; 666 (24.0\%), 5.0 - 9.9\%; 863 (31.2\%), $5.0 \%$ or more was $55.2 \%$ and $3.0 \%$ or more was $71.4 \%$.

respectively [12]. Successively, 6-years period analyses showed that the total weight reduction was $0.6 \mathrm{~kg}$ in the low-fat, $3.1 \mathrm{~kg}$ in the Mediterranean, and $1.7 \mathrm{~kg}$ in LCD group, respectively ( $p=0.01$ for all comparisons) [13].

Our nutritional guidance is one that has been standardized as a manual [14]. This is a modified training program, Self-Regulation Empowerment Program (SREP) in the educational region. SREP has been generally used by school professionals to empower adolescent students to engage in self-motivating cycles of learning in a positive mind.

From the standpoint of RDNs, there are many subjects to be advised, but no one has the same background. Because everyone is the only one, we cannot deal with a single pattern. When actually teaching, it is important to build a longterm trust relationship between patients and staffs. RDNs will always stayed close together on supporting the patient due to each character and situation.

As a matter of fact, they interviewed frequently for the desires of the subjects. They basically followed the behavioral science protocol. It is not ready-made clothing but tailor-made clothes. It seems to be their specialized skills and friendly hospitality that brought the excellent achievement for weight reduction.

In the United States, RDN is estimated as the nation's food and nutrition expert. According to the recent reports on RDN in the US, RDNs tend to use an intuitive eating approach more often than previous traditional weight management manners [15].

There are five stages that RDNs usually use during patient guidance [14].

I. Self-monitoring is to grasp its current state. Graphing the weight measurement data and fixing the meal record would be basically important, which has been called "Recording Diet".

II. Self-assessment of lifestyle and consideration of factors affecting lifestyle are necessary.

III. It is important to clarify what the motivation is, what purpose or hope is. Motivational processes include selfregulation of learning, such as selfefficacy beliefs, goal orientations, and 
Citation: Nakamura T, Kawashima T, Dobashi M, et al., "Effective Nutritional Guidance for Obesity by Low Carbohydrate Diet (LCD)", Asp Biomed Clin Case Rep, vol.2, no.s1: 16-21, 2019.

purposes of engagement [16].

IV. After the success of weight loss, consider what future goals will be with the reference of 5-step theory advocated by Maslow.

V. If the patient has a good result, he will feel confident, efficacy and selfevaluation. Our staffs can give cognitive interviewing, which would bring patients the ability to gathering information about the cognitive process and beliefs obtained by the experience [16].

A staff can establish a trust relationship with the patient by an appropriate distance and encouragement. With this repetition, the patient himself will also have a cyclical and selfregulation feedback loop [14].

There was a report of weight reduction study by an intensive lifestyle intervention (ILI) for the Look AHEAD (Action for Health in Diabetes) study [17]. They performed the ILI and diabetes support and education (DSE) interventions. Interventionists included registered dietitians, psychologists, and exercise specialists. They incorporated cognitive behavioral therapy, problem-solving, motivational interviewing, and cultural tailoring in their counseling [10]. Thus, these psychological approaches would be effective for weight reduction program.

This research has limitations. We have not compared two groups which are with or without the Empowerment Program. Then, the effectiveness of the Empowerment Program has not been clear. There is a comparative report of the empowerment program for chronic kidney disease (CKD), where differences in overall self-management and selfefficacy are investigated [18].

In summary, RDNs instructed 2773 obese patients. By LCD with $33 \%$ of carbohydrate, the weight reduction of more than $10 \%$ was $24.0 \%$, $5.0 \%$ or more was $55.2 \%$, and $3 \%$ or more was $71.4 \%$. This efficacy would be due to LCD, selfregulation empowerment program and reliability of nutritionists. These results suggest that current data would become a reference and fundamental data for weight reduction and contribute to future research development.

\section{Conflicts Of Interest}

The authors state that we do not have any conflicts of interest in this report.

\section{References}

[1] American Diabetes Association, "Pharmacologic Approaches to Glycemic Treatment: Standards of Medical Care in Diabetes-2018". Diabetes Care, vol.41, suppl.1: S73-S85, 2018.

[2] Sato J, Kanazawa A, Makita S, et al., "A randomized controlled trial of $130 \mathrm{~g} /$ day lowcarbohydrate diet in type 2 diabetes with poor glycemic control". Clin Nutr, vol.36, no.4: 9921000, 2017.

[3] Feinman RD, Pogozelski WK, Astrup A, et al., "Dietary carbohydrate restriction as the first approach in diabetes management: Critical review and evidence base". Nutrition, vol.31, no.1: 1-13, 2015.

[4] Tay J, Thompson CH, Luscombe-Marsh ND, et al., "Effects of an energy-restricted low carbohydrate, high unsaturated fat/low saturated fat diet versus a high-carbohydrate, low-fat diet in type 2 diabetes: A 2-year randomized clinical trial". Diabetes Obes Metab, vol.20, no.4: 85871, 2018.

[5] Meng Y, Bai H, Wang S, et al., "Efficacy of low carbohydrate diet for type 2 diabetes mellitus management: A systematic review and metaanalysis of randomized controlled trials". Diabetes Res Clin Pract, vol.131 : 124-31, 2017.

[6] Ebe K, Ebe Y, Yokota S, et al., "Low Carbohydrate diet (LCD) treated for three cases as diabetic diet therapy". Kyoto Medical Association Journal, vol.51: 125-29, 2004.

[7] Nakamura T, Bando H, "Low carbohydrate diet (LCD) has to be applied for nutritional therapy". The Journal of the Therapy, vol.91: 2858-59, 2009.

[8] Nakamura T, Bando $\mathrm{H}$, "Total analysis of weight reduction project in the orthopedic clinic". $12^{\text {th }}$ proceeding of Japan Anti-Aging Association: 2012.

[9] Bando H, Ebe K, Nakamura T, et al., "Low Carbohydrate Diet (LCD): Long and short-term effects and hyperketonemia". Glycative Stress 
Citation: Nakamura T, Kawashima T, Dobashi M, et al., "Effective Nutritional Guidance for Obesity by Low Carbohydrate Diet (LCD)", Asp Biomed Clin Case Rep, vol.2, no.s1: 16-21, 2019.

Research, vol.3, no.4: 193-204, 2016.

[10] West DS, Dilillo V, Bursac Z, et al., "Motivational Interviewing Improves Weight Loss in Women with Type 2 Diabetes". Diabetes Care, vol.30, no.5: 1081-87, 2007.

[11] Armstrong MJ, Mottershead TA, Ronksley $\mathrm{PE}$, et al., "Motivational interviewing to improve weight loss in overweight and/or obese patients: a systematic review and metaanalysis of randomized controlled trials". Obes Rev, vol.12, no.9: 709-23, 2011.

[12] Shai I, Schwarzfuchs D, Henkin Y, et al., "Weight loss with a low-carbohydrate, Mediterranean, or low-fat diet". N Engl J Med, vol.359, no.3: 229-41, 2008.

[13] Schwarzfuchs D, Golan R, Shai I, "Four-year follow-up after two-year dietary interventions". $N$ Engl J Med, vol.367, no.14: 1373-74, 2012.

[14] Cleary TJ, Zimmerman BJ, "Self-regulation empowerment program: A school-based program to enhance self-regulated and self- motivated cycles of student learning". Psychology in the Schools, vol.41, no.5: 537-50, 2004.

[15] Schaefer JT, Zullo MD, "US Registered Dietitian Nutritionists' Knowledge and Attitudes of Intuitive Eating and Use of Various Weight Management Practices". J Acad Nutr Diet, vol.117, no.9: 1419-28, 2017.

[16] Bembenutty $H$, "New directions for selfregulation of learning in postsecondary education". New Directions for Teaching and Learning, vol.2011, no.126: 117-24, 2011.

[17] The Look AHEAD Research Group, "Eightyear weight losses with an intensive lifestyle intervention: the look AHEAD study". Obesity, vol.22, no.1: 5-13, 2013.

[18] Lee SJ, "An empowerment program to improve self-management in patients with chronic kidney disease". Korean J Adult Nurs, vol.30, no.4: 426-36, 2018.

Keywords: Obesity; Low Carbohydrate Diet (LCD); Weight Reduction; Nutritional Guidance; SelfRegulation Empowerment Program (SREP); Registered Dietitian Nutritionist (RDN) 